\title{
Measures on compact HS spaces
}

\author{
by
}

Mirna D ž a mo n j a and Kenneth K u n e n (Madison, Wis.)

\begin{abstract}
We construct two examples of a compact, 0-dimensional space which supports a Radon probability measure whose measure algebra is isomorphic to the measure algebra of $2^{\omega_{1}}$. The first construction uses $\diamond$ to produce an S-space with no convergent sequences in which every perfect set is a $G_{\delta}$. A space with these properties must be both hereditarily normal and hereditarily countably paracompact. The second space is constructed under $\mathrm{CH}$ and is both HS and HL.
\end{abstract}

0. Introduction. All spaces considered here are Hausdorff. A perfect set is a non-empty closed set with no isolated points.

Suppose $X$ is compact and supports a Radon probability measure $\mu$ such that the measure algebra of $(X, \mu)$ is not separable; does this imply that $X$ can be mapped continuously onto $[0,1]^{\omega_{1}}$ ? This question is open in ZFC. In particular, Haydon asked whether such an implication might follow from something like MA $+\neg \mathrm{CH}$; see Fremlin [2] for more discussion. Under $\mathrm{CH}$, there is a counterexample which is, in addition, a compact Lspace (hereditarily Lindelöf (HL) but not hereditarily separable (HS)); see [4, $6]$. In this paper, we show that, assuming $\diamond$, there is another counterexample which is an S-space (HS, but not HL). The space also has the property that every perfect set is a $G_{\delta}$, whereas no point is a $G_{\delta}$. Also, assuming just $\mathrm{CH}$, we construct a third counterexample which is both HS and HL.

Neither of the above mentioned examples could be constructed in ZFC, since under MA $+\neg \mathrm{CH}$, there are neither compact L-spaces (Juhász) nor compact S-spaces (Szentmiklóssy) (see [8]). Furthermore, under MA + $\neg \mathrm{CH}$, the measure algebra of any compact HL (equivalently, HS) Radon measure space is separable (Fremlin [2]).

The following theorem details the properties of the S-space. The HS + HL example is a modification of the S-space, and is described in $\S 4$.

Authors supported by NSF Grant DMS-9100665. 
0.1. THEOREM. If $\diamond$ holds, then there is a compact, 0-dimensional, hereditarily separable space $X$ and a Radon probability measure $\mu$ on $X$ such that

1. $X$ has weight $\omega_{1}$, and every point in $X$ has character $\omega_{1}$.

2. There are no convergent sequences in $X$.

3. Every perfect subset of $X$ is a $G_{\delta}$ set.

4. The measure algebra of $(X, \mu)$ is isomorphic to the measure algebra of $2^{\omega_{1}}$ with the usual product measure.

We do not need to state in the Theorem that $X$ cannot be mapped onto $[0,1]^{\omega_{1}}$, since that follows from hereditary separability. Likewise, Part (1) of the Theorem implies that $X$ is not hereditarily Lindelöf, since in a hereditarily Lindelöf space, every point is a $G_{\delta}$. Also, (1) implies that the cardinality of $X$ is $2^{\omega_{1}}$.

Our proof is patterned after the result, announced by Fedorchuk in [1], that under $\diamond$, there is a compact S-space of size $2^{\omega_{1}}$, although we do not follow exactly the method indicated in [1]. By induction on $\alpha \leq \omega_{1}$, we shall construct a closed $X_{\alpha} \subseteq 2^{\alpha}$. If $\alpha<\beta$, then $X_{\alpha}$ will be the projection of $X_{\beta}$. The $X$ of the Theorem will be $X_{\omega_{1}}$. To make sure that the space is hereditarily separable, we shall use $\diamond$ to capture all candidates for an $\omega_{1}$ left-separated sequence. This method will also capture all $\omega$-sequences as well, so that Part (2) of Theorem 0.1 will essentially come for free. To guarantee Part (3), we use $\diamond$ a second time to control the perfect sets.

A complete probability measure $\mu$ on $X$ is said to be Radon if it is defined on the Borel sets and has the property that the measure of each Borel set is the supremum of the measures of its compact subsets. Since $X$ will be compact and 0-dimensional, we may define such a measure by its values on the clopen sets, as we now explain. The Baire sets in $X$ are the least $\sigma$-algebra containing the clopen sets, and the Borel sets in $X$ are the least $\sigma$-algebra containing the open sets. Suppose that $\mu$ is a finitely additive probability measure defined on the clopen subsets of $X$. Then, in the standard way, $\mu$ defines an outer measure, from which we define an extension of $\mu$ to a countably additive probability measure $\widehat{\mu}$ on some $\sigma$-algebra, $\mathcal{S}$. $\mathcal{S}$ certainly contains all Baire sets. It need not contain all Borel sets, but if it does, then $\widehat{\mu}$ is automatically a Radon measure.

So, why should all Borel sets be $\widehat{\mu}$-measurable? In an HL space (as in $\S 4$ ), this is trivial, since the Borel and Baire sets coincide. In fact, for any compact $X, X$ is HL iff all closed sets are $G_{\delta}$ sets iff all Borel sets are Baire. Also, for any compact $X$, a closed set is Baire iff it is a $G_{\delta}$. In our particular space, (1) says that each point is a non- $G_{\delta}$ and hence non-Baire. However, (4) implies that the measure algebra is non-atomic, so that points have measure 0 ; in particular, they are $\widehat{\mu}$-measurable. Now, suppose $K$ is any closed set, and let $I$ be the set of isolated points of $K$. By HS, $I$ is 
countable, and hence $\widehat{\mu}$-measurable (and of measure 0). By (2), either $I$ is finite and $K=I$ or $I$ is infinite and $K \backslash I$ is perfect, and hence a $G_{\delta}$ by (3). In either case, $K$ is the union of a measurable set and a Baire set, and hence measurable. A subtle point: of course, we must verify (4), or at least that $\widehat{\mu}$ gives points measure 0 , without needing that every Borel set is $\widehat{\mu}$-measurable; but we do that in $\S 1$.

Since the measure is determined by its values on the clopen sets, it is easily constructed by induction. When we construct $X_{\alpha}$, we also decide what the measure is of all its clopen subsets. Then when we get to $X=$ $X_{\omega_{1}}$, we will also have $\mu$ and hence $\widehat{\mu}$, and as we pointed out above, $\widehat{\mu}$ will almost automatically be a Radon measure. We still have to ensure that the resulting measure algebra is isomorphic to the measure algebra of $2^{\omega_{1}}$. To do that, we shall simply make it everywhere non-separable; the isomorphism will then follow by Maharam's Theorem [7]. However, doing this requires an additional complication on the construction of the $X_{\alpha}$, which makes verifying that we can get an S-space somewhat harder. We comment on this further at the end of $\S 3$.

We proceed by listing a number of requirements on the $X_{\alpha}$ and $\mu_{\alpha}$ which, if met, guarantee that the resulting $(X, \mu)$ satisfies Theorem 0.1 . In $\S 1$, we describe the basic requirements, which guarantee that $X$ will have every point of character $\omega_{1}$ and a Baire measure $\mu$ such that the resulting measure algebra is isomorphic to the measure algebra of $2^{\omega_{1}}$. In $\S 2$, we describe some additional requirements on the construction which will guarantee the rest of the properties of $X$. In $\S 3$, we verify that all the requirements of $\S \S 1,2$ can be simultaneously fulfilled, thus proving Theorem 0.1 ; we also explain there why a space with the properties of Theorem 0.1 must be hereditarily normal and hereditarily countably paracompact. In $\S 4$, we explain how to use $\mathrm{CH}$ and modify the construction to get a space which is both HS and HL.

There is a somewhat simpler class of compact S-spaces $[3,5,8]$ which require only $\mathrm{CH}$ to construct; but these have points of countable character, so we could not use them to prove Theorem 0.1 .

1. The basic stuff. For $\alpha \leq \beta$, define $\pi_{\alpha}^{\beta}: 2^{\beta} \rightarrow 2^{\alpha}$ by $\pi_{\alpha}^{\beta}(f)=f\lceil\alpha$. We shall choose $X_{\alpha}$ for $\alpha \leq \omega_{1}$ and $A_{\alpha}, B_{\alpha}$ for $\alpha<\omega_{1}$ so that:

R1.1. $X_{\alpha}$ is a closed subset of $2^{\alpha}$, and $\pi_{\alpha}^{\beta}\left(X_{\beta}\right)=X_{\alpha}$ whenever $\alpha<\beta$ $\leq \omega_{1}$.

R1.2. For $\alpha<\omega_{1}, A_{\alpha}$ and $B_{\alpha}$ are closed in $X_{\alpha}, A_{\alpha} \cup B_{\alpha}=X_{\alpha}$, and $X_{\alpha+1}=A_{\alpha} \times\{0\} \cup B_{\alpha} \times\{1\}$. Here, we identify $2^{\alpha+1}$ with $2^{\alpha} \times\{0,1\}$.

R1.3. For $n<\omega, X_{n}=A_{n}=B_{n}=2^{n}$. For $\alpha \geq \omega, A_{\alpha}$ and $B_{\alpha}$ have no isolated points. 
We remark that this may be viewed as an inductive construction. The choice of $A_{\alpha}$ and $B_{\alpha}$ determines $X_{\alpha+1}$ from $X_{\alpha}$, and, by R1.1, $X_{\gamma}$ is determined from the earlier $X_{\alpha}$ at limit $\gamma$. R1.3 avoids some trivialities; it implies that for $\alpha \geq \omega, X_{\alpha}$ has no isolated points; also, $X_{\omega}=2^{\omega}$.

We also choose $\mu_{\alpha}$ for $\alpha<\omega_{1}$ so that:

R1.4. $\mu_{\alpha}$ is a finitely additive probability measure on the clopen subsets of $X_{\alpha}$, and $\mu_{\alpha}=\mu_{\beta}\left(\pi_{\alpha}^{\beta}\right)^{-1}$ whenever $\alpha<\beta \leq \omega_{1}$.

Again, this may be viewed as an inductive construction. For limit $\gamma, \mu_{\gamma}$ is determined from the earlier $\mu_{\alpha}$. As described in the introduction, each $\mu_{\alpha}$ extends to a $\widehat{\mu}_{\alpha}$ on some $\sigma$-algebra, $\mathcal{S}_{\alpha}$, which contains all the Baire sets. For $\alpha<\omega_{1}$, the Baire and Borel sets coincide, but this need not hold for $\alpha=\omega_{1}$.

At a successor stage, in the case that $\widehat{\mu}_{\alpha}\left(A_{\alpha} \cap B_{\alpha}\right)>0$, there is some freedom in defining $\mu_{\alpha+1}$, in that the measure on $A_{\alpha} \cap B_{\alpha}$ can be distributed arbitrarily over $\left(A_{\alpha} \cap B_{\alpha}\right) \times\{0\}$ and $\left(A_{\alpha} \cap B_{\alpha}\right) \times\{1\}$. For the purpose of this paper, the equitable distribution will work. That is,

R1.5. For each $\alpha<\omega_{1}$, and each Borel $D \subseteq A_{\alpha} \cap B_{\alpha}, \widehat{\mu}_{\alpha+1}(D \times\{0\})=$ $\widehat{\mu}_{\alpha+1}(D \times\{1\})=\frac{1}{2} \widehat{\mu}_{\alpha}(D)$.

In terms of the clopen sets, this defines $\mu_{\alpha+1}$ from $\mu_{\alpha}$. Specifically, every clopen $K \subseteq X_{\alpha+1}$ can be written uniquely as $K=K_{0} \times\{0\} \cup K_{1} \times\{1\}$, where $K_{0}$ is a relatively clopen subset of $A_{\alpha}$ and $K_{1}$ is a relatively clopen subset of $B_{\alpha}$. Then $\mu_{\alpha+1}(K)=\widehat{\mu}_{\alpha}\left(K_{0}\right)+\widehat{\mu}_{\alpha}\left(K_{1}\right)-\frac{1}{2} \widehat{\mu}_{\alpha}\left(K_{0} \cap B_{\alpha}\right)-\frac{1}{2} \widehat{\mu}_{\alpha}\left(K_{1} \cap A_{\alpha}\right)$. In the special case that $K$ is the inverse projection of a clopen set $L \subset X_{\alpha}$, then $L=K_{0} \cup K_{1}$ and $K_{0} \cap B_{\alpha}=K_{1} \cap A_{\alpha}=K_{0} \cap K_{1}$, so $\mu_{\alpha+1}(K)=\widehat{\mu}_{\alpha}\left(K_{0}\right)+$ $\widehat{\mu}_{\alpha}\left(K_{1}\right)-\widehat{\mu}_{\alpha}\left(K_{0} \cap K_{1}\right)=\widehat{\mu}_{\alpha}\left(K_{0} \cup K_{1}\right)=\mu_{\alpha}(L)$. Thus, $\mu_{\alpha+1}=\mu_{\alpha}\left(\pi_{\alpha}^{\alpha+1}\right)^{-1}$. This shows that condition R1.4 gets preserved at successor stages. It is also easy to verify:

\subsection{LemmA. Requirements R1.1-1.5 imply that}

1. For $\alpha \leq \omega, \widehat{\mu}_{\alpha}$ is the usual product measure on $X_{\alpha}$.

2. For all $\alpha, \widehat{\mu}_{\alpha}$ gives each non-empty clopen set positive measure.

3. For all $\alpha \geq \omega, \widehat{\mu}_{\alpha}$ gives each point measure 0 .

R1.1-R1.5 do not prevent us from choosing $A_{\alpha} \cap B_{\alpha}$ to be of measure 0 (or even a singleton) for all $\alpha \geq \omega$. In that case, the measure algebra on $X=X_{\omega_{1}}$ would be isomorphic to the measure algebra of $2^{\omega}$. To prevent this, we shall demand

R1.6. For each $\alpha<\omega_{1}$ and each closed $J \subseteq X_{\alpha}$, if $\widehat{\mu}_{\alpha}(J)>0$, then there is a $\beta \geq \alpha$ such that $\widehat{\mu}_{\beta}\left(\left(\pi_{\alpha}^{\beta}\right)^{-1}(J) \cap A_{\beta} \cap B_{\beta}\right)>0$. 
1.2. Lemma. Requirements R1.1-1.6 imply that the measure algebra of $(X, \widehat{\mu})$ is isomorphic to the measure algebra of $2^{\omega_{1}}$ (with the usual product measure).

Pro of. Let $\mathcal{B}$ be the measure algebra; so, elements of $\mathcal{B}$ are equivalence classes of $\widehat{\mu}$-measurable subsets of $X$. For each $b \in \mathcal{B}$, let $\mathcal{B}\lceil b$ be the algebra $\{a \in \mathcal{B}: a \leq b\}$ (so, $b$ is the 1 of $\mathcal{B}\lceil b$ ). By Maharam's Theorem [7], the lemma will follow if we can show that for all $b \neq 0, \mathcal{B}\lceil b$ is not separable.

For $\alpha<\omega_{1}$, let $\mathcal{B}_{\alpha}$ be the subalgebra of $\mathcal{B}$ generated by all $\left(\pi_{\alpha}^{\omega_{1}}\right)^{-1}(H)$, for $H$ a Borel (= Baire) subset of $X_{\alpha}$. Then $\mathcal{B}$ is an ascending union, $\mathcal{B}=\bigcup_{\alpha} \mathcal{B}_{\alpha}$. If $\mathcal{B}\left\lceil b\right.$ were separable, we may fix $\alpha<\omega_{1}$ such that $b \in \mathcal{B}_{\alpha}$ and $\mathcal{B}\left\lceil b=\mathcal{B}_{\alpha}\left\lceil b\right.\right.$. Then, there is a closed $J \subseteq X_{\alpha}$ such that $\widehat{\mu}_{\alpha}(J)>0$ and $[J] \leq b$; applying R1.6 and R1.5 to this $J$ yields a contradiction.

The following requirement implies (actually is equivalent to) that every point in $X_{\omega_{1}}$ has character $\omega_{1}$.

R1.7. For each $\alpha<\omega_{1}$ and each $y \in X_{\alpha}$, there is a $\beta$ with $\alpha \leq \beta<\omega_{1}$ and $A_{\beta} \cap B_{\beta} \cap\left(\pi_{\alpha}^{\beta}\right)^{-1}\{y\} \neq \emptyset$.

1.3. Lemma. Requirements R1.1-1.7 imply that every point in $X_{\omega_{1}}$ has character $\omega_{1}$.

Proof. If $x \in X_{\omega_{1}}$ had countable character, then there would be an $\alpha<\omega_{1}$ such that $\left(\pi_{\alpha}^{\omega_{1}}\right)^{-1}\{x\lceil\alpha\}=\{x\}$. Applying R1.7 to $y=x\lceil\alpha$ yields a contradiction.

Requirements R1.1-R1.7 are consistent with having $A_{\alpha}=B_{\alpha}=X_{\alpha}$ for all $\alpha$, whence $X$ will simply be $2^{\omega_{1}}$ with the usual product measure. We cannot get an S-space unless $A_{\alpha} \cap B_{\alpha}$ is nowhere dense in $X_{\alpha}$ for all but countably many $\alpha$.

2. How to use $\diamond$. We begin with some notation on sequences. Suppose that $\vec{y}=\left\langle y_{\xi}: \xi<\beta\right\rangle$ is a $\beta$-sequence of distinct points in some space. Then a point $x$ is a limit point of $\vec{y}$ iff for all neighborhoods $U$ of $x, \exists \xi\left(y_{\xi} \in U\right.$ $\left.\wedge x \neq y_{\xi}\right)$. Now, suppose that $\vec{Y}=\left\langle Y_{\xi}: \xi<\beta\right\rangle$ is a $\beta$-sequence of disjoint sets. We shall call $x$ a strong limit point of $\vec{Y}$ iff for all neighborhoods $U$ of $x, \exists \xi\left(Y_{\xi} \subseteq U \wedge x \notin Y_{\xi}\right)$. This implies that however we choose points $y_{\xi} \in Y_{\xi}, x$ will be a limit point of $\left\langle y_{\xi}: \xi<\beta\right\rangle$.

For ordinals $\alpha<\beta$, if $\vec{f}=\left\langle f_{\xi}: \xi<\beta\right\rangle$ is a $\beta$-sequence in $2^{\beta}$, we shall use $\vec{f}\left\lceil\alpha\right.$ for the $\alpha$-sequence in $2^{\alpha},\left\langle f_{\xi}\lceil\alpha: \xi<\alpha\rangle\right.$. Assuming $\diamond$, we can fix $f^{\vec{\alpha}}$ for each $\alpha \in\left[\omega, \omega_{1}\right)$, such that $f^{\vec{\alpha}}$ is an $\alpha$-sequence in $2^{\alpha}$ and such that whenever $\vec{g}$ is an $\omega_{1}$-sequence in $2^{\omega_{1}},\left\{\alpha: \vec{g}\left\lceil\alpha=f^{\alpha}\right\}\right.$ is stationary.

For each $\beta \leq \omega_{1}$, we may postulate 
$\mathrm{R} 2.1(\beta)$. For all $\alpha \leq \beta$ : If the $f_{\xi}^{\alpha}$, for $\xi<\alpha$, are all distinct points in $X_{\alpha}, h \in X_{\beta}$, and $h\left\lceil\alpha\right.$ is a limit point of $f^{\alpha}$, then $h$ is a strong limit point of $\left\langle\left(\pi_{\alpha}^{\beta}\right)^{-1}\left(f_{\xi}^{\alpha}\right): \xi<\alpha\right\rangle$.

The reason that $\diamond$ is relevant to capturing sequences in $2^{\omega_{1}}$ is given by the following lemma, which states that some basic properties of such sequences reflect on a club (closed-unbounded set).

2.1. Lemma. Suppose that $\vec{f}$ is an $\omega_{1}$-sequence of distinct points in $2^{\omega_{1}}$. Then there is a club $C \subseteq \omega_{1}$ such that for all $\gamma \in C$,

1. The $f_{\xi}\lceil\gamma$ for $\xi<\gamma$ are all distinct.

2. If $\eta \geq \gamma$, then $f_{\eta} \uparrow \gamma$ is a limit point of $\vec{f} \uparrow \gamma$.

Proof. Let $\operatorname{Fn}(\gamma, 2)$ be the set of finite partial functions from $\gamma$ to 2, and, for $s \in \operatorname{Fn}(\gamma, 2)$, let $Z_{s}$ be the basic clopen subset of $2^{\gamma},\left\{f \in 2^{\gamma}: f \uparrow\right.$ $\operatorname{dom}(s)=s\}$. To achieve (1), make sure that whenever $\gamma \in C$,

$$
\forall \xi<\eta<\gamma \exists s \in \operatorname{Fn}(\gamma, 2)\left(f_{\xi} \in Z_{s} \wedge f_{\eta} \notin Z_{s}\right) .
$$

To achieve (2), make sure that whenever $\gamma \in C$ and $s \in \operatorname{Fn}(\gamma, 2)$,

$$
\sup \left\{\xi<\gamma: f_{\xi} \in Z_{s}\right\}<\gamma \Leftrightarrow \sup \left\{\xi<\omega_{1}: f_{\xi} \in Z_{s}\right\}<\gamma .
$$

In particular, if $\eta \geq \gamma, s \in \operatorname{Fn}(\gamma, 2)$, and $f_{\eta} \in Z_{s}$, then $\sup \left\{\xi<\omega_{1}: f_{\xi} \in\right.$ $\left.Z_{s}\right\} \geq \eta$, so there are unboundedly many $\xi<\gamma$ such that $f_{\xi} \in Z_{s}$.

2.2. Lemma. Assuming the requirements $\mathrm{R} 1.1-1.6$ and $\mathrm{R} 2.1\left(\omega_{1}\right), X$ is hereditarily separable.

Proof. If not, then let $\vec{f}$ be a left-separated $\omega_{1}$-sequence in $X$. Let $C$ be as in Lemma 2.1 and fix $\gamma \in C$ such that $\vec{f}\left\lceil\gamma=f^{\gamma}\right.$. Let $h=f_{\gamma}$. By Lemma 2.1, $h \uparrow \gamma$ is a limit point of $\vec{f} \uparrow \gamma, h$ is a strong limit point of $\left\langle\left(\pi_{\alpha}^{\omega_{1}}\right)^{-1}\left(f_{\xi}^{\gamma}\right): \xi<\gamma\right\rangle$, and hence a limit point of $\left\langle f_{\xi}: \xi<\gamma\right\rangle$, which is impossible if $\vec{f}$ is left-separated.

By a sequence we mean an $\omega$-sequence. One could ensure that the space has no convergent sequences by a similar use of $\diamond$ to capture $\omega$-sequences, but it turns out that this comes for free because of the way we captured $\omega_{1}$-sequences.

2.3. LemmA. Assuming R1.1-R1.7 and R2.1( $\left.\omega_{1}\right)$, there are no convergent sequences in $X$.

Proof. Suppose $\vec{x}$ is a discrete sequence in $X$ and $y$ is a limit point of $\vec{x}$. Let $\vec{f}$ be any $\omega_{1}$-sequence of points in $X$ such that $f_{n}=x_{n}$ for $n<\omega$ and $f_{\xi}(0) \neq y(0)$ for all $\xi \geq \omega$. Fix an $\alpha<\omega_{1}$ such that $\pi_{\alpha}^{\omega_{1}}(\vec{f})=f^{\vec{\alpha}}$ and 
note that $y\left\lceil\alpha\right.$ is a limit point of $f^{\vec{\alpha}}$. Then every point in $\left(\pi_{\alpha}^{\omega_{1}}\right)^{-1}(y\lceil\alpha)$ is a strong limit point of $\left\langle\left(\pi_{\alpha}^{\omega_{1}}\right)^{-1}\left(f_{\xi}^{\alpha}\right): \xi<\alpha\right\rangle$, and hence a limit point of $\left\langle f_{\xi}: \xi<\alpha\right\rangle$. Since we have separated the $f_{\xi}$ for $\xi \geq \omega$ away from $y$ at coordinate 0 , every point in $\left(\pi_{\alpha}^{\omega_{1}}\right)^{-1}(y\lceil\alpha)$ is a limit point of $\vec{x}$. By R1.7, $\left(\pi_{\alpha}^{\omega_{1}}\right)^{-1}(y\lceil\alpha)$ will not be a singleton, so $\vec{x}$ does not converge to $y$.

We shall now replace requirement $\mathrm{R} 2.1$ by a somewhat more complicated requirement on the $A_{\alpha}$ and $B_{\alpha}$, so that it will be clear that this is something which may be ensured during the inductive construction. First, note that there is no problem at limits.

2.4. LEMMA. If $\gamma$ is a limit and $\mathrm{R} 2.1(\beta)$ holds for all $\beta<\gamma$, then $\mathrm{R} 2.1(\gamma)$ holds as well.

However, there is a problem at successors. Suppose $\mathrm{R} 2.1(\beta)$ holds and $h \in X_{\beta+1}$. If $h \uparrow \beta \notin A_{\beta} \cap B_{\beta}$, then $\mathrm{R} 2.1(\beta+1)$ will hold at the point $h$, since at $h$, the projection $\pi_{\beta}^{\beta+1}$ is locally a homeomorphism. But, if $h \uparrow \beta \in A_{\beta} \cap B_{\beta}$, then R2.1 $(\beta+1)$ will fail, for example, if for some $\alpha<\beta$, the $f_{\xi}^{\alpha}$, for $\xi<\alpha$, are all distinct points in $X_{\alpha}$ and each $\left(\pi_{\alpha}^{\beta}\right)^{-1}\left(f_{\xi}^{\alpha}\right)$ meets both $A_{\beta}$ and $B_{\beta}$. To avoid this, we assume

R2.2. For all $\alpha \leq \beta<\omega_{1}$ : If the $f_{\xi}^{\alpha}$, for $\xi<\alpha$, are all distinct points in $X_{\alpha}, h \in A_{\beta} \cap B_{\beta}$, and $h$ is a strong limit point of $\left\langle\left(\pi_{\alpha}^{\beta}\right)^{-1}\left(f_{\xi}^{\alpha}\right): \xi<\alpha\right\rangle$, and $U$ is any neighborhood of $h$, then $\exists \xi\left(\left(\pi_{\alpha}^{\beta}\right)^{-1}\left(f_{\xi}^{\alpha}\right) \subseteq U \wedge\left(\pi_{\alpha}^{\beta}\right)^{-1}\left(f_{\xi}^{\alpha}\right) \cap A_{\beta}=\emptyset\right)$ and $\exists \xi\left(\left(\pi_{\alpha}^{\beta}\right)^{-1}\left(f_{\xi}^{\alpha}\right) \subseteq U \wedge\left(\pi_{\alpha}^{\beta}\right)^{-1}\left(f_{\xi}^{\alpha}\right) \cap B_{\beta}=\emptyset\right)$.

2.5. Lemma. Assuming the requirements $\mathrm{R} 1.1-1.6$ and $\mathrm{R} 2.2, X$ is hereditarily separable and has no convergent sequences.

P r o of. By induction on $\beta$, verify $\mathrm{R} 2.1(\beta)$, and then apply Lemmas 2.2 and 2.3.

We may now regard R2.1 as obsolete, having been replaced by R2.2.

We must still make sure that each perfect set $F$ becomes a $G_{\delta}$. To do this, we arrange for $F$ to become $\left(\pi_{\alpha}^{\omega_{1}}\right)^{-1}(H)$ for some $\alpha<\omega_{1}$ and some perfect $H$ in $X_{\alpha}$.

If $K$ is a closed subset of a space $Z$ and $g: Z \rightarrow Y$, we shall call $g$ irreducible on $K$ iff $g(L)$ is a proper subset of $g(K)$ for all proper closed subsets $L$ of $K$. Note that if $F$ is a closed subset of $Z$, then $F \subseteq g^{-1}(g(F))$; and if $g$ is irreducible on $g^{-1}(g(F))$, then $F=g^{-1}(g(F))$.

So, we shall arrange that for every perfect $F$, there will be an $\alpha<\omega_{1}$ such that $\pi_{\alpha}^{\omega_{1}}$ is irreducible on $\left(\pi_{\alpha}^{\omega_{1}}\right)^{-1}\left(\pi_{\alpha}^{\omega_{1}}(F)\right)$. Then $F=\left(\pi_{\alpha}^{\omega_{1}}\right)^{-1}\left(\pi_{\alpha}^{\omega_{1}}(F)\right)$ will be a $G_{\delta}$. As in the usual inductive construction of the projective cover, irreducibility of $\pi_{\alpha}^{\omega_{1}}$ can be guaranteed during the inductive construction by a condition on the $A_{\alpha}$ and $B_{\alpha}$, which we now explain. If $A \subseteq H \subseteq Y$, where $Y$ is any space, $A$ is called regular closed in $H$ iff $A=\operatorname{cl}_{H}\left(\operatorname{int}_{H}(A)\right)$; 
here $\mathrm{cl}_{H}$ and $\operatorname{int}_{H}$ denote the closure and interior operators relative to the subspace $H$. We call $A, B \subseteq H$ complementary regular closed sets in $H$ iff they are each regular closed in $H, A \cup B=H$, and $A \cap B$ is nowhere dense in $H$; this implies that $A \cap B$ is the common boundary of $A$ and $B$ in $H$, and that the natural projection from $A \times\{0\} \cup B \times\{1\}$ onto $H$ is irreducible.

2.6. LemmA. Suppose $H$ is closed in $X_{\alpha}$ and, for all $\beta \geq \alpha, A_{\beta} \cap$ $\left(\pi_{\alpha}^{\beta}\right)^{-1}(H)$ and $B_{\beta} \cap\left(\pi_{\alpha}^{\beta}\right)^{-1}(H)$ are complementary regular closed subsets of $\left(\pi_{\alpha}^{\beta}\right)^{-1}(H)$. Then $\pi_{\alpha}^{\omega_{1}}$ is irreducible on $\left(\pi_{\alpha}^{\omega_{1}}\right)^{-1}(H)$.

Proof. By induction on $\beta \leq \omega_{1}$, show that $\pi_{\alpha}^{\beta}$ is irreducible on $\left(\pi_{\alpha}^{\beta}\right)^{-1}(H)$.

Using $\diamond$, we can fix closed sets $F_{\alpha}$ in $2^{\alpha}$, for $\alpha<\omega_{1}$, such that for every closed $F$ in $2^{\omega_{1}},\left\{\alpha: \pi_{\alpha}^{\omega_{1}}(F)=F_{\alpha}\right\}$ is stationary. Once we have constructed $X_{\alpha}$, where $\omega \leq \alpha<\omega_{1}$, we shall define $Q_{\alpha}$ to be $F_{\alpha}$ if $F_{\alpha}$ is perfect and a subset of $X_{\alpha}$, and set $Q_{\alpha}=X_{\alpha}$ otherwise. So, $Q_{\alpha}$ is always a perfect subset of $X_{\alpha}$.

We now note that the property of not having isolated points is reflected on a club:

2.7. Lemma. If $F$ is a perfect subset of $2^{\omega_{1}}$, then there is a club $C$ such that for all $\alpha \in C, \pi_{\alpha}^{\omega_{1}}(F)$ is a perfect subset of $2^{\alpha}$.

R2.3. If $\alpha \geq \omega$ then for all $\beta \geq \alpha$

a. $A_{\beta} \cap B_{\beta} \cap\left(\pi_{\alpha}^{\beta}\right)^{-1}\left(Q_{\alpha}\right)$ is nowhere dense in $\left(\pi_{\alpha}^{\beta}\right)^{-1}\left(Q_{\alpha}\right)$.

b. $A_{\beta} \cap\left(\pi_{\alpha}^{\beta}\right)^{-1}\left(Q_{\alpha}\right)$ and $B_{\beta} \cap\left(\pi_{\alpha}^{\beta}\right)^{-1}\left(Q_{\alpha}\right)$ are complementary regular closed subsets of $\left(\pi_{\alpha}^{\beta}\right)^{-1}\left(Q_{\alpha}\right)$.

Actually, R2.3a is redundant, given $\mathrm{R} 2.3 \mathrm{~b}$, but when we actually verify in $\S 3$ that $\mathrm{R} 2.3$ can be accomplished, it will be easier to handle R2.3a before considering $\mathrm{R} 2.3 \mathrm{~b}$.

It is clear from the preceding lemmas that

2.8. Lemma. Assuming R1.1-R1.3 and R2.3, all perfect subsets of $X$ are $G_{\delta}$

3. Putting it together. We are now done with the proof of Theorem 0.1, assuming that the construction can be made to meet all of our requirements. Examining them, it appears that only R1.6, R1.7, R2.2, and R2.3 are non-trivial; the rest just detail how the final $X$ and $\mu$ are completely determined by the choice of $A_{\alpha}$ and $B_{\alpha}$ for $\omega \leq \alpha<\omega_{1}$. Let $S_{\alpha}=A_{\alpha} \cap B_{\alpha}$. Then R1.6 and R1.7 only involve what happens eventually, and only involve the $S_{\alpha}$; they ensure that every closed set of positive measure gets split (so the measure algebra will be non-separable) and that every point eventually gets split (so points get uncountable character). R2.3a must be met at every 
stage $\alpha$, but only involves $S_{\alpha}$. Finally, R2.2 and R2.3b must also be met at each stage, and actually involve $A_{\alpha}$ and $B_{\alpha}$, so we consider them last, after fixing $S_{\alpha}$.

Partition $\omega_{1} \backslash \omega$ into two disjoint uncountable sets, $E V E N$ and $O D D$. We shall handle R1.6 in EVEN and R1.7 in ODD. Applying CH, for $\beta \in E V E N$, choose $\delta_{\beta} \leq \beta$ and a closed $J_{\beta} \subseteq 2^{\delta_{\beta}}$ so that for each $\alpha<\omega_{1}$ and each closed $J \subseteq 2^{\alpha}$, there is a $\beta \geq \alpha$ such that $\beta \in E V E N$ and $\delta_{\beta}=\alpha$ and $J_{\beta}=J$. Again applying $\mathrm{CH}$, for $\beta \in O D D$, choose $\delta_{\beta} \leq \beta$ and $p_{\beta} \in 2^{\delta_{\beta}}$ so that for each $\alpha<\omega_{1}$ and each $p \in 2^{\alpha}$, there is a $\beta \geq \alpha$ such that $\beta \in O D D$ and $\delta_{\beta}=\alpha$ and $p_{\beta}=p$.

These choices are made ahead of time, before constructing the $X_{\alpha}$. Once $X_{\beta}$ is constructed, let $K_{\beta}=\left(\pi_{\delta_{\beta}}^{\beta}\right)^{-1}\left(J_{\beta}\right)$ if $J_{\beta} \subseteq X_{\delta_{\beta}}$, and set $K_{\beta}=X_{\beta}$ otherwise. Use $N_{\alpha}$ to denote the set $\left\{f_{\xi}^{\alpha}: \xi<\alpha\right\}$.

Now, determine $S_{\beta}$ as follows.

For $\beta \in E V E N$, choose $S_{\beta}$ so that

$\Sigma 1$. If $\widehat{\mu}_{\beta}\left(K_{\beta}\right)>0$, then $\widehat{\mu}_{\beta}\left(K_{\beta} \cap S_{\beta}\right)>0$.

$\Sigma 2$. For all $\alpha \in(\omega, \beta], S_{\beta} \cap\left(\pi_{\alpha}^{\beta}\right)^{-1}\left(Q_{\alpha}\right)$ is nowhere dense in $\left(\pi_{\alpha}^{\beta}\right)^{-1}\left(Q_{\alpha}\right)$.

$\Sigma 3$. For all $\alpha \in(\omega, \beta], S_{\beta} \cap\left(\pi_{\alpha}^{\beta}\right)^{-1}\left(N_{\alpha}\right)=\emptyset$.

It is clear that $\Sigma 1$ will guarantee requirement R1.6 and $\Sigma 2$ will guarantee R2.3a for $\beta \in E V E N$. $\Sigma 3$ will make it possible to choose $A_{\beta}$ and $B_{\beta}$ later. First, we must see that such a choice of $S_{\beta}$ is possible. For each $\alpha \in(\omega, \beta]$, choose a countable $D_{\alpha} \subseteq\left(\pi_{\alpha}^{\beta}\right)^{-1}\left(Q_{\alpha}\right)$ which is dense in $\left(\pi_{\alpha}^{\beta}\right)^{-1}\left(Q_{\alpha}\right)$. For each $\alpha \in(\omega, \beta]$, let $E_{\alpha}=N_{\alpha} \cap X_{\alpha}$. Let $L=K_{\beta}$ if $\widehat{\mu}_{\beta}\left(K_{\beta}\right)>0$; set $L=X_{\beta}$ otherwise. Let $M=L \backslash \bigcup_{\alpha \in[\omega, \beta)}\left(D_{\alpha} \cup\left(\pi_{\alpha}^{\beta}\right)^{-1}\left(E_{\alpha}\right)\right) . \quad M$ is a positive measure set minus a countable union of measure 0 sets (since by Lemma 1.1, points have measure 0 ). Thus, $M$ has positive measure, and we may choose $S_{\beta}$ to be any closed subset of $M$ of positive measure.

For $\beta \in O D D$, choose $S_{\beta}$ as follows: If $p_{\beta} \in X_{\delta_{\beta}}$, choose $S_{\beta}$ to be any singleton from $\left(\pi_{\alpha}^{\beta}\right)^{-1}\left\{p_{\beta}\right\}$. If not, set $S_{\beta}$ to be any singleton. It is clear that this choice will guarantee requirement R1.7. It also guarantees R2.3a for $\beta \in O D D$, since $\left(\pi_{\alpha}^{\beta}\right)^{-1}\left(Q_{\alpha}\right)$ has no isolated points.

Proof of Theorem 0.1. As noted above, we are done provided we can show that at each stage $\beta$ we can can satisfy R2.2 and R2.3b. We already have $S_{\beta}$, and we must find $A_{\beta}$ and $B_{\beta}$. Fix a strictly decreasing sequence of clopen sets in $X_{\beta},\left\langle V_{n}: n \in \omega\right\rangle$, such that $V_{0}=X_{\beta}$ and $\bigcap_{n \in \omega} V_{n}=S_{\beta}$. If $\phi: \omega \rightarrow \omega$ is any strictly increasing function with $\phi(0)=0$, we may set $A_{\beta}=S_{\beta} \cup \bigcup_{n}\left(V_{\phi(2 n)} \backslash V_{\phi(2 n+1)}\right)$ and $B_{\beta}=S_{\beta} \cup \bigcup_{n}\left(V_{\phi(2 n+1)} \backslash V_{\phi(2 n+2)}\right)$. With any choice of $\phi, A_{\beta} \cap B_{\beta}=S_{\beta}$, and the "trivial" conditions R1.2 and R1.3 are met. We shall show that if $\phi$ grows "fast enough", both R2.2 and R2.3b will be met - essentially by the same argument. 
To handle R2.3b, fix, for each $\alpha \leq \beta$, a countable dense subset $T_{\alpha}$ of $S_{\beta} \cap$ $\left(\pi_{\alpha}^{\beta}\right)^{-1}\left(Q_{\alpha}\right)$. Since $S_{\beta} \cap\left(\pi_{\alpha}^{\beta}\right)^{-1}\left(Q_{\alpha}\right)$ is nowhere dense in $\left(\pi_{\alpha}^{\beta}\right)^{-1}\left(Q_{\alpha}\right)$, there is for each element $t$ of $T_{\alpha}$ a sequence $\overrightarrow{s_{t}}$ of distinct points in $\left(\pi_{\alpha}^{\beta}\right)^{-1}\left(Q_{\alpha}\right) \backslash S_{\beta}$ converging to $t$. Note that R2.3b will hold if for all $\alpha \leq \beta$ and $t \in T_{\alpha}$, both $A_{\beta} \backslash B_{\beta}$ and $B_{\beta} \backslash A_{\beta}$ contain infinitely many elements of $\overrightarrow{s_{t}}$.

To simplify the notation, we re-index all the $\left\langle\left(\pi_{\alpha}^{\beta}\right)^{-1}\left(f_{\xi}^{\alpha}\right): \xi<\alpha\right\rangle$ for $\alpha<\beta$ which are relevant to R2.2, as well as all the sequences $\overrightarrow{s_{t}}$ just chosen. We then have countably many sequences, $\vec{Y}^{i}(i \in \omega)$. Each $\overrightarrow{Y^{i}}$ is a sequence of disjoint closed sets (identifying each of the sequences of points $\overrightarrow{s_{t}}$ with the corresponding sequence of singletons). Since the notion of strong limit point does not depend on the order type of the sequence, we may as well assume that each $Y^{i}$ is an $\omega$-sequence, so $\overrightarrow{Y^{i}}=\left\langle Y_{j}^{i}: j<\omega\right\rangle$. If $i \in \omega$ and $U$ is a clopen subset of $X_{\beta}$, let $R(i, U)$ be the assertion that for each point $h \in S_{\beta}$, if $h$ is a strong limit point of $\overrightarrow{Y^{i}}$, and $U$ is a neighborhood of $h$, then there are infinitely many $n$ such that

$$
\exists j\left(Y_{j}^{i} \subseteq U \cap\left(V_{\phi(2 n)} \backslash V_{\phi(2 n+1)}\right)\right) \wedge \exists j\left(Y_{j}^{i} \subseteq U \cap\left(V_{\phi(2 n+1)} \backslash V_{\phi(2 n+2)}\right)\right) .
$$

Then both R2.2 and R2.3b will hold if $R(i, U)$ holds for each $i, U$. To accomplish this we shall, for each $i, U$, find a $\psi_{i, U}: \omega \rightarrow \omega$ such that $R(i, U)$ holds whenever $\phi(l+1) \geq \psi_{i, U}(\phi(l))$ for all but finitely many $l$; this will be sufficient to be able to define $\phi$, since there are only countably many $i$ and $U$.

So, fix $i$ and $U$. We must find $\psi=\psi_{i, U}$ such that for each point $h \in S_{\beta}$, if $h$ is a strong limit point of $Y^{i}$, and $U$ is a neighborhood of $h$, then for each $m, \psi(m)>m$ and

$$
\exists j\left(Y_{j}^{i} \subseteq U \cap\left(V_{m} \backslash V_{\psi(m)}\right)\right) .
$$

Now, fix $m$, and assume that there is some $h \in U \cap S_{\beta}$ such that $h$ is a strong limit point of $Y^{i}$, since otherwise our condition is vacuous. Then, fix $j$ with $Y_{j}^{i} \subseteq\left(U \cap V_{m}\right)$ and $h \notin Y_{j}^{i}$. Note that $Y_{j}^{i} \cap S_{\beta}=\emptyset$; this is obvious when $\overrightarrow{Y^{i}}$ comes from one of the $\overrightarrow{s_{t}}$ (since none of the points in $\overrightarrow{s_{t}}$ is in $S_{\beta}$ ), or when $\beta \in O D D$ (since then $S_{\beta}=\{h\}$ ). When $\beta \in E V E N$, it follows from item $\Sigma 3$ in our choice of $S_{\beta}$ above. By compactness, there must be an $r>m$ such that $Y_{j}^{i} \cap V_{r}=\emptyset$, and we choose such an $r$ for $\psi(m)$.

If we only wanted to construct an S-space, then we could have made all the $S_{\beta}$ singletons. That would simplify the proof - especially in the above discussion of R2.2, where the $U$ could always be one of the $V_{n}$, and $\phi$ could be chosen by a simple diagonal argument. Making all the $S_{\beta}$ singletons would also force the measure algebra to be separable.

It is not clear whether one could do the above construction under $\mathrm{CH}$, without $\diamond$. Even without the measure, it is already a well-known open ques- 
tion whether $\mathrm{CH}$ alone implies the existence of a compact S-space of size greater than $\omega_{1}$.

In answer to a question of Peter Nyikos, we now show that our space is hereditarily normal. It is also hereditarily countably paracompact. Furthermore, both of these properties follow from the properties of the space stated in Theorem 0.1.

3.1. Theorem. Suppose that $X$ is compact, $X$ has no uncountable discrete subsets and no convergent sequences, and every perfect subset of $X$ is a $G_{\delta}$. Then $X$ is hereditarily normal and hereditarily countably paracompact.

The proof of Theorem 3.1 seems somewhat simpler if we follow M. E. Rudin and express our properties in terms of "shrinkings" of countable (or finite) covers.

3.2. LemmA. A space $Z$ is normal and countably paracompact iff for all $\beta \leq \omega$ and all open covers of $Z,\left\{U_{n}: n<\beta\right\}$, there are closed $H_{n} \subseteq U_{n}$ such that $\left\{H_{n}: n<\beta\right\}$ covers $Z$.

From Lemma 3.2, it is easy to prove the following well-known result.

3.3. LEMMA. Suppose that the space $Z$ has a locally finite cover by closed sets, each of which is normal and countably paracompact in its relative topology. Then $Z$ is normal and countably paracompact.

Now, note, as we did in $\S 0$, that the hypotheses on $X$ in Theorem 3.1 imply that every infinite closed subset of $X$ is the union of a perfect set, which we denote now by $\operatorname{ker}(X)$, and a countable set of isolated points. If $X$ is finite, let $\operatorname{ker}(X)=\emptyset$. Then $\operatorname{ker}(X)$ is always a $G_{\delta}$.

3.4. LEMmA. If $X$ is as in Theorem 3.1 and $p \in X$, then $X \backslash\{p\}$ is normal and countably paracompact.

Proof. Applying Lemma 3.2, let $\beta \leq \omega$ and let $\left\{U_{n}: n \leq \beta\right\}$ be an open cover of $X \backslash\{p\}$. If $p \in \operatorname{ker}\left(X \backslash U_{n}\right)$ for each $n$, then $\{p\}$ would be the intersection of countably many $G_{\delta}$ sets and hence a $G_{\delta}$, which is impossible, since $p$ would then be the limit of a convergent sequence. So, fix $i$ such that $p$ is isolated in $X \backslash U_{i}$. Let $U_{i}^{\prime}=U_{i} \cup\{p\}$, and let $U_{n}^{\prime}=U_{n}$ for $n \neq i$. Then the $U_{n}^{\prime}$ form an open cover of $X$, which is compact, so we may shrink the $U_{n}^{\prime}$ to closed $H_{n}^{\prime}$ in $X$ and then let $H_{n}=H_{n}^{\prime} \cap X \backslash\{p\}$.

Since normality and countable paracompactness are hereditary to closed subsets, we now know that whenever $K$ is closed in $X, K \backslash\{p\}$ is normal and countably paracompact. Actually, the proof of 3.4 shows that $K \backslash\{p\}$ is countably compact, although we do not need that fact here.

Proof of Theorem 3.1. It is enough to prove that $X \backslash H$ is normal and countably paracompact whenever $H$ is closed. Since $\operatorname{ker}(H)$ is a $G_{\delta}$, 
we may find a countable locally finite (in $X \backslash H$ ) cover of $X \backslash H$ by closed (in $X \backslash H)$ sets, where each set in the cover is of the form $K$ or $K \backslash\{p\}$, with $K$ closed in $X$. Thus, by Lemma 3.3, $X \backslash H$ is normal and countably paracompact.

Actually, $X \backslash H$ is countably compact iff $\operatorname{ker}(H)$ is clopen.

4. Getting the HS+HL space. To make the space HL also, we simply make every closed set a $G_{\delta}$-not just the perfect sets. This time, we only need $\mathrm{CH}$.

4.1. Theorem. If $\mathrm{CH}$ holds, then there is a compact 0-dimensional, hereditarily separable and hereditarily Lindelöf space $X$ and a Radon probability measure $\mu$ on $X$ such that the measure algebra of $(X, \mu)$ is isomorphic to the measure algebra of $2^{\omega_{1}}$ with the usual product measure. $X, \mu$ also have the property that all measure 0 sets are second countable in their relative topology.

To prove this theorem, we modify the construction from the previous proof. HS +HL is guaranteed by a similar use of irreducible maps, as explained by the next lemma.

4.2. Lemma. Assume just R1.1 ( $X$ is closed in $2^{\omega_{1}}$ and $X_{\alpha}$ is its projection on $\left.2^{\alpha}\right)$. Then

(a) $X$ is $H L$ iff for all closed $H \subseteq X$, there is an $\alpha<\omega_{1}$ for which $H=\left(\pi_{\alpha}^{\omega_{1}}\right)^{-1}\left(\pi_{\alpha}^{\omega_{1}}(H)\right)$.

(b) $X$ is $H L+H S$ iff for all closed $H \subseteq X$, there is an $\alpha<\omega_{1}$ for which $\pi_{\alpha}^{\omega_{1}}$ is irreducible on $\left(\pi_{\alpha}^{\omega_{1}}\right)^{-1}\left(\pi_{\alpha}^{\omega_{1}}(H)\right)$.

Pr o of. Part (a) follows from the fact that HL is equivalent to all closed sets being $G_{\delta}$ sets (for compact $X$ ).

For the $\Leftarrow$ of Part (b), irreducibility implies that $H=\left(\pi_{\alpha}^{\omega_{1}}\right)^{-1}\left(\pi_{\alpha}^{\omega_{1}}(H)\right)$, so $X$ is HL by Part (a). Since the irreducible preimage of a separable space is separable, all closed subsets of $X$ are separable, which implies that $X$ is HS, since $X$ is first countable.

For the $\Rightarrow$ of Part (b), assume $X$ is HS+HL. Let $H$ be closed in $X$, and let $E$ be a countable dense subset of $H$. Applying Part (a), we may fix $\alpha<\omega_{1}$ such that $H=\left(\pi_{\alpha}^{\omega_{1}}\right)^{-1}\left(\pi_{\alpha}^{\omega_{1}}(H)\right)$ and $\{e\}=\left(\pi_{\alpha}^{\omega_{1}}\right)^{-1}\left(\pi_{\alpha}^{\omega_{1}}(e)\right)$ for all $e \in E$. If $F$ is any closed subset of $H$ such that $\pi_{\alpha}^{\omega_{1}}(F)=\pi_{\alpha}^{\omega_{1}}(H)$, then for each $e \in E, \pi_{\alpha}^{\omega_{1}}(e) \in \pi_{\alpha}^{\omega_{1}}(F)$, so $e \in F$; hence $E \subseteq F$, so $F=H$. Thus, $\pi_{\alpha}^{\omega_{1}}$ is irreducible on $H=\left(\pi_{\alpha}^{\omega_{1}}\right)^{-1}\left(\pi_{\alpha}^{\omega_{1}}(H)\right)$.

Now, to prove Theorem 4.1, we delete R1.7 (which gave points uncountable character), and replace it by R4.1 below, which will have just the opposite effect. We also delete R2.1-R2.2, which relied on $\diamond$. Requirement R2.3, 
which guaranteed irreducibility of maps, remains the same as it was, but the $Q_{\alpha}$ will have a different meaning.

Using $\mathrm{CH}$, choose, for all countable $\alpha \geq \omega$, an ordinal $\delta_{\alpha} \leq \alpha$ and a Borel set, $J_{\alpha} \subseteq 2^{\delta_{\alpha}}$, so that for all $\gamma<\omega_{1}$ and each Borel set $J \subseteq 2^{\gamma}$, there is an $\alpha \geq \gamma$ such that $\delta_{\alpha}=\gamma$ and $J_{\alpha}=J$.

Once we have defined $X_{\alpha}$, where $\omega \leq \alpha<\omega_{1}$, we define the following subsets of $X_{\alpha}$ :

$$
\begin{aligned}
& C_{\alpha}=\left(\pi_{\delta_{\alpha}}^{\alpha}\right)^{-1}\left(J_{\alpha}\right) \text { if } J_{\alpha} \subseteq X_{\delta_{\alpha}} ; C_{\alpha}=\emptyset \text { otherwise. } \\
& K_{\alpha}=C_{\alpha} \text { if } C_{\alpha} \text { is closed; } K_{\alpha}=X_{\alpha} \text { otherwise. } \\
& Q_{\alpha}=K_{\alpha} \backslash \bigcup\left\{O: O \text { is open } \wedge \mu_{\alpha}\left(K_{\alpha} \cap O\right)=0\right\} . \\
& N_{\alpha}=\left(K_{\alpha} \backslash Q_{\alpha}\right) \cup C_{\alpha} \text { if } \mu_{\alpha}\left(C_{\alpha}\right)=0 ; N_{\alpha}=\left(K_{\alpha} \backslash Q_{\alpha}\right) \text { otherwise. }
\end{aligned}
$$

Then we require

R4.1. For any $\beta \geq \alpha \geq \omega, A_{\beta} \cap B_{\beta} \cap\left(\pi_{\alpha}^{\beta}\right)^{-1}\left(N_{\alpha}\right)=\emptyset$.

4.3. Lemma. Assume the requirements R1.1-R1.6, R2.3, and R4.1. Let $H$ be a closed subset of $X$. Then there is an $\alpha<\omega_{1}$ such that $\pi_{\alpha}^{\omega_{1}}$ is irreducible on $\left(\pi_{\alpha}^{\omega_{1}}\right)^{-1}\left(\pi_{\alpha}^{\omega_{1}}(H)\right)$.

Proof. For each $\gamma<\omega_{1}$, let $H_{\gamma}=\pi_{\gamma}^{\omega_{1}}(H)$. Then the $\mu_{\gamma}\left(H_{\gamma}\right)$ form a non-increasing sequence of real numbers, so we may fix a $\gamma<\omega_{1}$ such that for all $\alpha \geq \gamma, \mu_{\alpha}\left(H_{\alpha}\right)=\mu_{\gamma}\left(H_{\gamma}\right)$. Now fix an $\alpha \geq \gamma$ such that $\delta_{\alpha}=\gamma$ and $J_{\alpha}=H_{\gamma}$. Then $K_{\alpha}=C_{\alpha}=\left(\pi_{\gamma}^{\alpha}\right)^{-1}\left(H_{\gamma}\right)$. Then $H_{\alpha}$ is a closed subset of $K_{\alpha}$ with the same measure, so $Q_{\alpha} \subseteq H_{\alpha} \subseteq K_{\alpha}$. Now, $\pi_{\alpha}^{\omega_{1}}$ is irreducible on $\left(\pi_{\alpha}^{\omega_{1}}\right)^{-1}\left(Q_{\alpha}\right)$ (by R2.3b and Lemma 2.6), and $\pi_{\alpha}^{\omega_{1}}$ is $1-1$ on $\left(\pi_{\alpha}^{\omega_{1}}\right)^{-1}\left(H_{\alpha} \backslash Q_{\alpha}\right)$ (by R4.1). Thus, $\pi_{\alpha}^{\omega_{1}}$ must be irreducible on $\left(\pi_{\alpha}^{\omega_{1}}\right)^{-1}\left(H_{\alpha}\right)$ as well.

Including the $C_{\alpha}$ in $N_{\alpha}$ ensures that all measure 0 sets will be second countable.

Proof of Theorem 4.1. By Lemmas 4.2 and 4.3, we are done, provided all the requirements can be met. But the proof of this is exactly as for Theorem 0.1, except that now $O D D=\emptyset$ and $E V E N=\omega_{1} \backslash \omega$.

We remark that the space in [6] also needed only $\mathrm{CH}$ and satisfied everything in Theorem 4.1 except being HS. The construction in [6] did not use irreducibility, but rather established HL by making the measure 0 ideal and the first category ideal coincide; this was accomplished by taking $A_{\beta}=X_{\beta}$ for all $\beta$. The requirement of separability forces there to be a first-category set of measure 1 .

\section{References}

[1] V. V. Fedorchuk, On the cardinality of hereditarily separable compact spaces, Dokl. Akad. Nauk SSSR 222 (1975), 302-305 (in Russian). 
[2] D. Fremlin, Consequences of Martin's Axiom, Cambridge University Press, 1984.

[3] A. Hajnal and I. Juhász, On first countable non-Lindelöf $S$-spaces, in: Colloq. Math. Soc. János Bolyai 10, North-Holland, 1975, 837-852.

[4] R. Haydon, On dual $L^{1}$-spaces and injective bidual Banach spaces, Israel J. Math. 31 (1978), 142-152.

[5] I. Juhász, K. Kunen and M. E. Rudin, Two more hereditarily separable nonLindelöf spaces, Canad. J. Math. 28 (1976), 998-1005.

[6] K. Kunen, A compact L-space under CH, Topology Appl. 12 (1981), 283-287.

[7] D. Maharam, On homogeneous measure algebras, Proc. Nat. Acad. Sci. U.S.A. 28 (1942), 108-111.

[8] J. Roitman, Basic $S$ and L, in: Handbook of Set-Theoretic Topology, K. Kunen and J. Vaughan (eds.), North-Holland, 1984, 295-326.

UNIVERSITY OF WISCONSIN

MADISON, WISCONSIN 53706

U.S.A.

E-mail: DZAMONJA@MATH.WISC.EDU and KUNEN@CS.WISC.EDU 